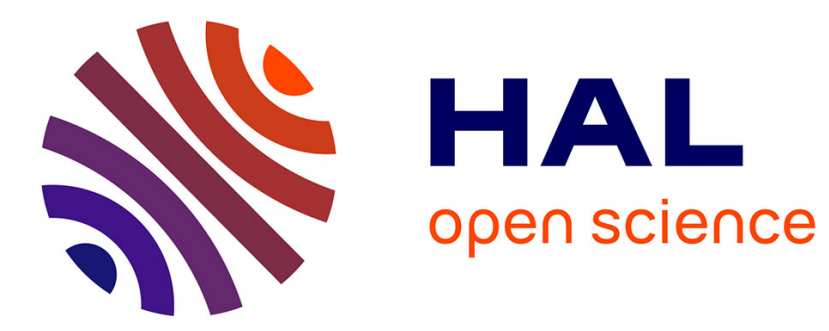

\title{
AGIR EN SITUATION DE DISCRÉDIT
}

Catherine Delcroix

\section{To cite this version:}

Catherine Delcroix. AGIR EN SITUATION DE DISCRÉDIT. Migrations Société, 2011, Migrations, racismes, résistances, 23 (133), pp.81-93. hal-01285020

\section{HAL Id: hal-01285020 \\ https://hal.science/hal-01285020}

Submitted on 8 Mar 2016

HAL is a multi-disciplinary open access archive for the deposit and dissemination of scientific research documents, whether they are published or not. The documents may come from teaching and research institutions in France or abroad, or from public or private research centers.
L'archive ouverte pluridisciplinaire HAL, est destinée au dépôt et à la diffusion de documents scientifiques de niveau recherche, publiés ou non, émanant des établissements d'enseignement et de recherche français ou étrangers, des laboratoires publics ou privés. 


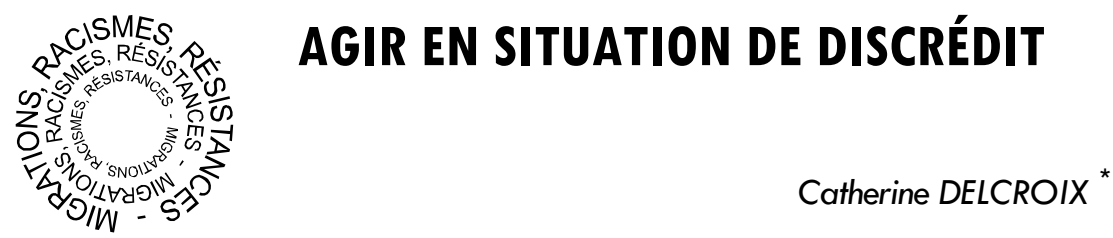

Au cours de notre carrière, nous avons effectué plusieurs recherches sur diverses catégories sociales en situation de précarité ${ }^{1}$. C'est ainsi que nous nous sommes rendu compte peu à peu que chaque fois que les gens qui vivent ce type de situation doivent agir pour tenter de l'améliorer ils se heurtent - outre la faiblesse de leurs ressources - à un type de difficulté supplémentaire: on ne leur fait a priori aucun crédit au sens premier, théorique du terme.

C'est le cas, par exemple, du jeune Français habitant une cité HLM stigmatisée quand il cherche un premier emploi : il a une mauvaise adresse. Si en plus il est issu de l'immigration... C'est le cas aussi du couple d'immigrés qui cherche un logement et celui du Portugais, ouvrier du bâtiment, qui demande auprès d'une banque un premier crédit pour se mettre à son compte. D'une manière générale, c'est le cas de tous les membres d'une catégorie qui se trouvent du mauvais côté des rapports de domination : toute situation de dominé-e est simultanément une situation de discrédit, une situation de discrédité-e a priori sur lequel (laquelle) pèsent toutes sortes de stéréotypes négatifs : en mettant les choses au pire, cette personne sera spontanément perçue comme passive, réfléchissant peu, sans capacité

* Professeur de sociologie, Laboratoire Cultures et Sociétés en Europe (CNRS - Université de Strasbourg).

1. Ce chapitre s'appuie sur les résultats de plusieurs recherches menées en France et de manière comparative dans six pays européens. Tout d'abord, une recherche financée par la Caisse nationale d'allocations familiales consacrée aux Enjeux prioritaires et types de conduites des familles populaires face à la précarité qui nous a permis de rencontrer dans la durée (1994-1998) plus de 30 familles vivant dans un même quartier. En 2000, nous avons mené une étude pour l'Institut des hautes études de la sécurité intérieure (IHESI) dans deux quartiers d'une ville de la région parisienne sur les stratégies familiales de prévention construites pour prévenir les risques de passage à la prédélinquance d'enfants âgés de 9 à 13 ans. Entre 1999 et 2003, nous avons été responsable de l'équipe française qui travaillait sur le thème du Household in precarity. Case histories from deprived neighbourhoods in six European countries, projet européen Built Environment : weather scenarios for investigation of impacts and extremes (BETWIXT) sous la direction de Daniel BertauX pour la Commission européenne. Entre 2005 et 2008, nous avons rencontré les membres de 40 familles vivant dans des cités HLM à Dreux et à Vernouillet pour qu'ils évaluent les effets des politiques de la ville et plus particulièrement du dispositif de renouvellement urbain dont leurs quartiers faisaient l'objet sur leur vie et celle de leurs enfants. II s'agissait d'une étude financée par l'Agence nationale pour la rénovation urbaine (ANRU). 
stratégique, instable, cherchant à vivre de prestations d'assistance plutôt que d'un emploi...

Or, quand on est dans une telle situation, on doit justement se battre simplement pour survivre, pour ne pas se laisser entraîner vers l'exclusion. Toutefois, non seulement ces personnes ont beaucoup moins de ressources objectives mobilisables - moins de capital économique, culturel, social mais en plus le discrédit qui les frappe en tant que membres d'une "catégorie sociale" discréditée rend beaucoup plus difficile la gestion de leur vie dans la durée (leurs cours d'action) ${ }^{2}$. Comme chacun de nous, elles ont besoin des autres pour mener à bien leurs projets, mais les esprits et les portes se ferment spontanément devant elles, qu'il s'agisse de ceux de l'entrepreneur qui recrute, du propriétaire de logement qui cherche un locataire sérieux ou du banquier qui pourrait accorder un crédit, tandis que ces mêmes portes et esprits vont s'ouvrir devant toute autre personne qui, à ressources objectives égales, ne souffre pas, elle, de discrédit.

Les personnes qui sont victimes de discrédit en raison par exemple de la couleur de leur peau, de leur nom, de leur adresse dans un quartier stigmatisé, de tel ou tel handicap apparent doivent donc accomplir des efforts beaucoup plus grands pour arriver au même résultat que celles qui ne le sont pas. En outre, ces efforts supplémentaires sont voués par nature à rester inaperçus aux yeux des membres d'autres milieux sociaux, qui ne vivent pas la même situation et n'en ont aucune idée. Même la plupart des sociologues - à part ceux qui ont étudié de près la vie des gens en situation de précarité - n'imaginent pas cette somme d'efforts supplémentaires. II y a comme une invisibilité des conséquences concrètes du discrédit. Ce sont ces deux idées que nous voudrions d'abord exemplifier avant de remonter en généralité.

\section{Domination et discrédit}

Des penseurs classiques de la sociologie et de la science politique tels que Karl Marx, Max Weber, Antonio Gramsci, ou encore Pierre Bourdieu ou Alain Touraine, ont établi que les sociétés occidentales sont structurées par des rapports de domination de différents types, dont les principaux sont les rapports de classe, les rapports de genre ou

2. Nous employons l'expression "cours d'action" pour désigner une séquence d'actions se déroulant dans une temporalité moyenne ou longue, dont la cohérence et la continuité découlent d'un projet visant soit à atteindre un objectif donné, soit à prévenir l'occurrence d'un événement négatif. 
"rapports sociaux de sexe", et les rapports de racisation spécifiques à l'histoire de chaque société. Ces rapports de domination engendrent des distributions très inégales des ressources matérielles et symboliques ${ }^{3}$. Pour ce qui est de l'action autonome, la question qui nous intéresse ici est l'inégale - et parfois très inégale — répartition des ressources qu'engendrent des conditions très contrastées. Du côté des "dominants", l'abondance en ressources matérielles, culturelles, sociales et symboliques ainsi que l'accès direct ou médiatisé à des ressources de pouvoir facilitent considérablement l'action. Du côté des "dominés", qu'on appellera ici discrédités, c'est l'inverse : la faiblesse des ressources de tout ordre, le manque d'autonomie dans les postes de travail, l'impossibilité d'accéder à des ressources de pouvoir rendent l'action autonome très difficile. Elle restreint considérablement l'horizon temporel et la possibilité d'élaborer des plans à long terme.

Nous proposons le terme "discrédit", car par son étymologie même il indique un déficit de crédibilité. Erving Goffman l'emploie dans un passage de Stigmate pour désigner toute personne qui II possède un attribut qui le rend différent des autres membres de [sa] catégorie [...], et aussi moins attrayant, qui, à l'extrême, fait de lui quelqu' un d'intégralement mauvais, ou dangereux, ou sans caractère. Ainsi diminué à nos yeux, il cesse d'être pour nous une personne accomplie et ordinaire, et tombe au rang d'individu vicié, amputé. Un tel attribut constitue un stigmate, surtout si le discrédit qu'il entraîne est très large [...]. II représente un désaccord particulier entre les identités sociales virtuelle et réelle $1{ }^{4}$.

Erving Goffman entend désigner ici les personnes qu'un handicap physique distingue, "I rend différentes des autres membres de [leur] catégorie II. Nous entendons cependant élargir ici le sens qu'il donne au terme "discrédit". Pour nous, il désigne en effet un processus de stigmatisation touchant toutes les personnes appartenant à une "catégorie" donnée du simple fait qu'elles en sont membres. Ce sont donc des catégories de perception collective, des catégories symboliques qui isolent "arbitrairement" (et politiquement) une caractéristique particulière, par exemple le handicap, mais aussi la position dans les rapports de production, le sexe, l'ethnicité... et la chargent de significations péjoratives, d'attributs présupposés, de préjugés stéréotypés. Ces catégories symboliques ont leur propre cycle historique : elles apparaissent dans des contextes historiques

3. Cf. PFEFFERKORN, Roland, Inégalités et rapports sociaux: rapports de classes, rapports de sexes, Paris : Éd. La Dispute, 2007, $412 \mathrm{p}$.

4. GOFFMAN, Erving, Stigmate : les usages sociaux du handicap, Paris : Éd. de Minuit, 1975, $175 \mathrm{p}$. (voir pp. 12-13). 
et politiques précis ; à leur apogée elles seront même considérées comme des catégories objectives, puis elles peuvent tomber en désuétude ou devenir moins fréquentes, comme ce fut le cas dans la société française pour les catégories de "fille-mère", "prolétaire", "Auvergnat" ou "Nord-Africain".

Pour autant, a-t-on raison d'en conclure que les "discrédités" en sont réduits à la passivité de l'agir et du penser? Que font-ils pour lever ce présupposé de passivité et d'incompétence?

\section{Poids du discrédit et résistance}

Prenons les étudiants d'une promotion de futurs ingénieurs appelés à trouver un stage dans une entreprise. Ce stage, obligatoire, leur permettra de terminer leur parcours d'études et d'obtenir leur diplôme. Parmi eux il y en a deux qui ont eu jusque-là les meilleures notes aux examens : Julien et Mohammed ${ }^{6}$.

Julien a 22 ans et il est fils d'un entrepreneur français. Dès que le moment est venu d'entamer les démarches pour chercher un stage, il demande à son père des adresses de professionnels susceptibles de l'engager comme stagiaire. Les personnes renseignées connaissent et respectent le père de Julien. Les rendez-vous sont obtenus facilement. Quand le jeune homme rencontre son futur responsable de stage, il bénéficie du crédit que lui apporte son identité héritée. Ses capacités à trouver rapidement un lieu de stage parfait lui valent les félicitations de ses enseignants, qui le donnent en exemple aux autres étudiants de la promotion : voici quelqu'un d'actif, de performant, et donc porteur d'avenir.

Mohammed, fils d'un migrant venu du Mali pour travailler comme ouvrier chez Renault, est français, âgé de 22 ans, comme Julien, et aussi un très bon étudiant. Mohammed se met aussi vite que Julien à chercher le stage indispensable à l'obtention de son diplôme. Mais, contrairement à Julien, pour trouver les lieux et les possibilités de stage, il est obligé de faire une recherche d'informations beaucoup plus longue, sans appui personnel, et d'essuyer nombre de refus.

Mohammed est tout de suite confronté à la nécessité de se rendre crédible pour obtenir la ressource indispensable à l'obtention de son diplôme :

5. Cf. NOIRIEL, Gérard, Immigration, antisémitisme et racisme en France, $X I X^{\ell}-X X^{e}$ siècle : discours publics, humiliations privées, Paris : Éd. Fayard, 2007, 717 p.

6. II s'agit ici de deux "personnages" construits à partir des cas que nous avons rencontrés au fil de nos enquêtes. 
le stage. Il doit accepter de partir des catégorisations d'autrui qui, en fonction de la couleur de sa peau, de sa "culture" supposée étrangère (alors qu'il n'en est rien) et de son origine de classe, le typifient ${ }^{7}$ comme a priori incapable d'assumer les responsabilités d'un futur cadre ou de ne pas être en mesure de "se faire accepter" par son futur environnement professionnel. Pour faire accepter son identité réelle il est en quelque sorte sommé de réduire l'écart entre celle-ci, qu'il porte en lui et qu'il revendique, et son identité attribuée par d'autres. II accepte alors de travailler gratuitement sans avoir le statut de stagiaire pour faire la preuve de ses capacités. Mais cette période de mise à l'épreuve retarde beaucoup le moment du démarrage officiel de son stage, ce qui l'oblige à redoubler. Les enseignants en concluent que si Mohammed a certes de grandes aptitudes scolaires, il est incapable de trouver son chemin dans le monde professionnel. Au discrédit qui frappe collectivement les enfants d'immigrés s'ajoute désormais celui qui lui est personnellement attaché. Tant que les obstacles (liés au discrédit) qui sont responsables de son échec restent invisibles (pas même imaginés), c'est à lui qu'en sera attribuée la responsabilité personnelle.

Si son directeur ou sa directrice de stage et de mémoire avait pris le temps de suivre pas à pas le cours d'action entrepris par Mohammed, il/elle aurait fini par prendre la mesure de la somme d'efforts qu'il lui a fallu faire. Quelle est l'évaluation la plus juste du mérite d'un étudiant? Celle qui se fonde sur le seul résultat ou celle qui prend en compte le chemin parcouru?

Au cours de son année de redoublement Mohammed doit trouver un employeur qui accepte de le prendre en stage ; c'est sa dernière chance. Il se donne d'abord pour objectif de convaincre son/sa professeur de se porter garant de son aptitude à assumer des responsabilités professionnelles et de l'accompagner dans sa démarche. II s'adresse aussi aux autres membres de l'équipe pédagogique et aux autres étudiants. II se crée un réseau de personnes capables de lui donner des informations, des conseils et qui attesteront de ses efforts pour trouver un stage.

Un cours d'action donné comme celui poursuivi par Mohammed est-il stratégique? II sera considéré comme tel par la théoricienne Lucy Suchman, par exemple, si ses efforts sont adéquats, si les moyens qu'il mobilise sont bien choisis, si une réflexion cohérente accompagne le cours de son action, s'il a mis en œuvre ce qu'elle appelle un " plan d'action ". Dans son ouvrage Plans and situated action, Lucy Suchman critique une conception abstraite

7. Cf. WATIER, Patrick, Une introduction à la sociologie compréhensive, Belval : Éd. Circé, 2002, 182 p. 
de l'action stratégique comme suivant les étapes d'un plan déterminé à l'avance par l'acteur. Elle rappelle que, dans la réalité, un cours d'action situé est un processus qui s'inscrit dans des contextes chargés d'incertitude et de contingences ${ }^{8}$. II faut donc considérer les plans que font les acteurs non pas tant comme des programmes à suivre pas à pas, mais comme des ressources guidant leur cours d'action, qui comprend nécessairement une part plus ou moins grande d'adaptation et d'improvisation.

Dans le cours d'action de Mohammed, la part des contingences, des reformulations des "stratégies", est souvent très grande, car il n'a que peu de contrôle sur son environnement et peu de ressources. Cela ne signifie pas qu'il ne cherche pas à "garder le cap".

II nous semble important de maintenir l'idée qu'il existe, pour un acteur donné à un moment donné, une situation objective. Certes, il ou elle est loin d'en percevoir et d'en connaître tous les tenants et les aboutissants, les contraintes et les possibilités cachées; sa perception initiale peut même en être fort éloignée. Mais c'est le travail de recherche d'informations effectué par l'acteur ainsi que ses premiers efforts pour aller vers un but qui constituent précisément les moyens de faire apparaître les caractéristiques objectives de sa situation.

On peut - et sans doute on doit - inclure dans ces caractéristiques la dimension intersubjective. Par exemple, le discrédit, qui pèse si fortement sur les demandes de coopération d'acteurs appartenant à des "catégories" (discursives) discréditées, est un phénomène intersubjectif ; il n'en reste pas moins qu'il prend parfois l'aspect d'un véritable mur, par exemple dans la recherche d'un stage pour un étudiant issu d'une famille de migrants, notamment non fortunée. Mais la référence à une "situation objective" dont les caractéristiques se découvrent progressivement au fil du cours d'action nous semble indispensable à une approche sociologique à visée généralisante. II ne s'agit pas d'exiger de l'acteur qu'il se comporte comme s'il avait une connaissance parfaite de son environnement (par exemple ici une connaissance parfaite du marché de l'emploi). On sait depuis les travaux de James $G$. March et Herbert A. Simon que les acteurs ne possèdent que des informations très incomplètes sur les contextes de leur action et quils agissent donc dans le cadre d'une "I rationalité limitée $11^{9}$.

8. Cf. SUCHMAN, Lucy, Plans and situated action: the problem of human-machine interaction, Cambridge : Cambridge University Press, 1987, $203 \mathrm{p}$.

9. MARCH, James G. ; SIMON, Herbert A., Les organisations, Paris : Éd. Dunod, 1965, 253 p. 
Michel Crozier et Erhard Friedberg synthétisent "I les résultats de toute une série d'études de décision au sein de grandes organisations " de la façon suivante : " L'être humain est incapable d'optimiser. Sa liberté et son information sont trop limitées pour qu'il y parvienne. Dans un contexte de rationalité limitée, il décide de façon séquentielle et choisit pour chaque problème qu'il a à résoudre la première solution qui correspond pour lui à un seuil minimal de satisfaction $1{ }^{10}$.

S'il en est ainsi des cadres dans les grandes organisations, des hauts fonctionnaires ou des entrepreneurs, cela doit être d'autant plus vrai pour les personnes en situation de relative précarité. Leur information est beaucoup plus limitée, et une bonne part de leur action consiste précisément ò rechercher cette précieuse ressource ${ }^{11}$.

De plus, le fait d'appartenir à des catégories en situation de discrédit ne produit pas seulement un stigmate qui a des effets négatifs sur l'estime de soi, mais il s'agit d'un handicap quand on veut développer un cours d'action. En effet, le capital symbolique aide à gagner la confiance - le crédit — d'autres acteurs dont l'aide est indispensable pour faire avancer le développement de son cours d'action.

Les difficultés répétées rencontrées par Mohammed — qui demeurent fort peu perceptibles dans leur véritable nature tant qu'on n'a pas soimême fait l'expérience du discrédit — atteignent l'estime de soi, la confiance en la réussite de ses projets et finalement les capacités de mobilisation, autrement dit le "moral" des acteurs victimes de discrédit. Julien, au contraire de Mohammed, a obtenu son diplôme et est entré sur le marché de l'emploi sûr de lui-même, prêt à s'engager complètement dans la vie professionnelle. L'impact du discrédit et des discriminations qui en découlent sur la subjectivité des individus est très fort. C'est pourquoi, pour un individu, le soutien des membres de son groupe familial, et particulièrement de ses frères et sœurs, constitue dans cette situation une ressource capitale.

À côté des contraintes, les situations sont aussi faites de ressources, qui sont de deux types : les ressources extérieures à l'individu et ses

10. CROZIER, Michel; FRIEDBERG, Erhard, L'acteur et le système: les contraintes de l'action collective, Paris : Éd. du Seuil, 2007, 500 p. (voir p. 54).

11. Un éducateur de quartier, à propos des habitants des "cités" lyonnaises, fait de la capacité à rechercher de l'information, éminemment variable d'une personne à l'autre, l'un des trois attributs de ce qu'il appelle la "puissance sociale", les deux autres étant la participation à des réseaux de solidarité actifs et la capacité à mettre en œuvre des savoir-faire pour résoudre les problèmes. Voir DELAPIERRE, Gilbert, "La puissance sociale des pères", Bulletin de la Société lyonnaise pour l'enfance et l'adolescence, 1993, pp. 20-23. 
ressources propres. Au nombre des ressources extérieures on peut compter les droits politiques et sociaux, les possibilités offertes par les marchés - de la formation, de l'emploi, du logement, etc. - les institutions publiques telles que l'Éducation nationale, les agences de protection sociale. La gratuité de l'enseignement et le système de bourses ont permis à Mohammed d'atteindre le niveau élevé de formation qui est le sien.

Pour ce qui est des ressources propres à l'individu, il convient de distinguer, d'un côté, les ressources objectives accumulées ou héritées, que Pierre Bourdieu appelle des " capitaux " économique, culturel, social et symbolique, et de l'autre les ressources de pouvoir liées à l'occupation d'une place d'autorité dans une institution disposant de ressources collectives : les personnes comme Mohammed sont en général dépourvues tant des premières que des secondes. Mais ce ne sont pas là les seules dont elles disposent.

En effet, ces hommes et ces femmes portent en eux-mêmes un autre type de ressources. Toutes ces expériences, qui sont faites au sein d'une sphère privée en continuité avec la sphère publique, aboutissent à ce que nous appelons des ressources subjectives spécifiques : compétences de compréhension, de création de liens intersubjectifs, de communication et de résolution de problèmes. Bien entendu, les expériences à elles seules ne suffisent pas; encore faut-il qu'elles aient été réfléchies, mises à distance, comparées pour qu'elles puissent s'intégrer aux ressources subjectives de la personne. Les " compétences pratiques " et les " compétences discursives " définies par Anthony Giddens en font par exemple partie ${ }^{12}$. Plus généralement encore, il faut entendre par ressources subjectives les énergies physiques, mentales et morales qu'un individu développe à un moment donné de son existence ainsi que ses savoirs et savoirfaire qui lui permettent de mobiliser à bon escient ses énergies, voire celles de ses proches, pour répondre à ses besoins et réaliser ses projets.

Mohammed est encouragé et aidé financièrement par sa famille qui lui assure toujours le gîte et le couvert, réduisant ainsi la nécessité pour lui de travailler pour payer ses études. II peut ainsi persévérer sans perdre patience : quand les ressources sont limitées, cette mobilisation familiale est souvent indispensable. II finit par obtenir un stage très intéressant qui lui permet d'être ensuite embauché. Son effort individuel a aussi été rendu possible par l'action collective d'étudiants placés comme lui en situation

12. GIDDENS, Anthony, La constitution de la société : éléments de la théorie de la structuration, Paris : Presses universitaires de France, 1987, 474 p. 
de discrédit et qui ne trouvaient pas de stage ou se faisaient exploiter. Ceux-ci ont protesté pour obtenir d'être reconnus comme des acteurs légitimes et ils ont participé à un mouvement qui peu à peu a pris une ampleur nationale.

On pourrait s'étonner de notre choix d'exemplifier les difficultés qu'il y a à agir en situation de discrédit sur le cas d'un étudiant brillant issu de l'immigration. Mais nous avons pris cet exemple à dessein, parce qu'il permet une comparaison avec un autre étudiant tout aussi brillant, mais bénéficiant au contraire de ce "crédit" qui facilite la réalisation d'un projet d'une façon tellement naturelle qu'elle en devient invisible. Nous avons aussi choisi cet exemple parce qu'il inclut la figure du professeur, qui incarne ici le phénomène d'aveuglement aux conditions concrètes de l'action des personnes victimes de discrédit, aveuglement "naturel" qui est la norme puisque les difficultés spécifiques que rencontre l'agir en situation de discrédit sont vouées à rester invisibles pour autrui.

\section{Un exemple inattendu de discrédit de genre}

Subir le poids du discrédit n'est pas réservé aux seules personnes vivant des situations de précarité. Nous proposons ici un autre exemple : il porte sur la difficulté, pour des pères de famille toutes origines sociales confondues, d'obtenir la garde de leur enfant en cas de séparation du couple. Nous nous fondons ici sur une enquête réalisée en 1990 pour la Caisse nationale des allocations familiales (CNAF) sur les conséquences du divorce sur la relation père-enfant ${ }^{13}$. Une enquête quantitative menée par l'Institut national d'études démographiques (INED) sur l'enfance avait montré à l'époque que $52 \%$ des pères n'avaient plus que des contacts épisodiques avec leur enfant après un divorce ou une séparation $^{14}$. Cette découverte était presque fortuite - ce n'était pas le but de l'enquête - et le questionnaire ne permettait pas d'expliquer les causes de ce phénomène, raison pour laquelle la CNAF nous avait commandé une enquête qualitative par études de cas. À l'époque - fin des années 1980 - les jugements de divorce attribuaient dans $85 \%$ des cas la garde de l'enfant à la mère et dans $8 \%$ à une grand-

13. Cf. BERTAUX, Daniel ; DELCROIX, Catherine, "La fragilisation du rapport père/enfant. Une enquête auprès des pères divorcés", Recherches et Prévisions, $\mathrm{n}^{\circ} 21$, septembre 1990, pp. 65-74; BERTAUX, Daniel ; DELCROIX, Catherine, Des pères face au divorce: la fragilisation du lien paternel, Paris : CNAF, Espaces et Familles, $\mathrm{n}^{\circ} 17$, juillet 1991,110 p.

14. Cf. LERIDON, Henri ; VILLENEUVE-GOKALP, Catherine, "Entre père et mère", Population \& Sociétés, $\mathrm{n}^{\circ} 220$, janvier 1988, $4 \mathrm{p}$. 
mère ou à une tante. Les pères ne l'obtenaient que dans moins de $7 \%$ des cas, alors qu'ils étaient bien plus nombreux à la demander, mais la loi ne permettait pas la garde partagée ou alternée.

En interrogeant des pères de toutes les catégories socioprofessionnelles, ainsi d'ailleurs que leurs ex-conjointes et d'autres personnes-témoins, nous avons peu à peu pris conscience du formidable discrédit qui touchait ces hommes, quelle que soit leur condition sociale, en tant qu'éducateurs de leur enfant. On ne leur faisait pas confiance sur ce point, même s'ils avaient une situation professionnelle confortable ou un excellent niveau d'éducation. Nous avons interrogé aussi des magistrats du tribunal de première instance, à qui la loi conférait tout pouvoir pour décider à qui confier la garde de l'enfant. Assez curieusement, aussi bien les magistrats masculins, généralement d'âge mûr, que les jeunes magistrates féministes convergeaient pour confier la garde à la mère, les premiers par conservatisme - " la famille est la cellule de base de notre société ; je tiens à protéger ce qu'il en reste... " - les secondes par conviction féministe progressiste : "Ce sont les hommes qui détiennent tous les leviers de pouvoir, alors je ne veux pas enlever aux femmes le seul qui leur reste ! II. La situation des pères non mariés en voie de séparation était encore plus difficile, puisqu'il leur fallait l'autorisation de la mère de leur enfant pour pouvoir partager avec elle l'autorité parentale.

Nous avons rencontré des pères qui voulaient absolument obtenir la garde de leur enfant, et nous avons été témoin des énormes efforts qu'ils devaient faire à l'époque, y compris quand ils étaient issus de la bourgeoisie, pour tenter de convaincre le magistrat ou la magistrate qu'ils sauraient parfaitement s'organiser pour élever seul leur enfant. C'était le cas d'Alexandre : "I'ai compris à la première audience que le juge, qui n'était pas très âgé, ne comprenait rien aux rapports des parents aux enfants. Chaque fois qu'il avait une question à poser, il la posait à ma femme, parce que cela lui paraissait évident que je n'y connaissais rien. C'était insupportable. C'est à des petites choses comme celles-là qu'on comprend que tout est faussé. Quand le juge s'est adressé cinq fois à votre femme pour lui demander plusieurs choses, vous comprenez qu'il pense que cela ne vous concerne pas. Je savais quelle allait être la décision $1{ }^{15}$. Nous avons même interrogé un père qui avait enlevé sa fille par désespoir. Comme quoi on peut être du bon côté du manche des rapports de domination et, sur le point précis de l'éducation d'un enfant, être quand même victime de discrédit, ce qui entrâne un

15. BERTAUX, Daniel ; DELCROIX, Catherine, La fragilisation du rapport père/enfant : une enquête sociologique, Paris : CNAF - Maison des sciences de l'homme, 1990, 293 p. (voir p. 238). 
surcroît considérable d'efforts pour parvenir à réaliser un objectif qui, pour les mères à l'époque, s'obtenait très facilement.

Nous avons montré précédemment, avec l'exemple de Mohammed, que la nécessité d'affronter le poids du discrédit conduit parfois à une mobilisation pour tenter de le lever collectivement : ici le mouvement des étudiants stagiaires de sa promotion, qui s'est étendu au niveau national. Pour ce qui est des pères séparés, nous avons observé l'action collective d'associations comme l'Association pour la défense des intérêts des divorcés hommes et de leurs enfants mineurs (DIDHEM), dont nous avons rencontré l'un des dirigeants qui était psychanalyste. Ils organisaient des conférences, diffusaient un bulletin et contactaient des parlementaires. Une fédération d'associations (le Mouvement de la condition paternelle) a fini par obtenir une modification de la loi qui, depuis le 4 mars 2002, autorise la garde partagée et la résidence alternée.

\section{Levée du poids du discrédit et action collective}

Au début des années 1990, nous avons aussi dirigé une rechercheaction menée dans un quartier populaire de Nantes. Un groupe d'éducateurs d'un club de prévention nous avait demandé de les aider à construire une enquête, qu'ils tenaient à faire eux-mêmes, sur les pères maghrébins du quartier, pour comprendre pourquoi ils n'exerçaient pas un contrôle plus strict sur leurs fils adolescents. Ces éducateurs découvrirent, en recueillant les récits de vie de quelques-uns de ces pères - souvent d'anciens militants - qu'ils s'étaient déjà regroupés de manière informelle pour aider leurs enfants à s'insérer et éviter ainsi des dérives. L'enquête a déclenché des dynamiques d'interaction entre le réseau informel des pères immigrés — que le discours des médias discréditait à travers des expressions telles que "parents démissionnaires ", " pères socialement invalidés $\| . .$. - le groupe d'éducateurs spécialisés et des réseaux de jeunes du quartier ; ces dynamiques ont duré plusieurs années. L'expérience nous a permis non seulement de constater de près les effets concrets du discrédit, mais aussi de suivre en temps réel un cours d'action collective qui a mené ces 57 pères à se constituer en association : l'Association culturelle des musulmans de Nantes-Nord (ACMNN). Après avoir été légitimée par les éducateurs auprès du pouvoir municipal et des habitants du quartier, cette association, qui compte aujourd'hui près de 400 pères, continue à mettre en œuvre un très grand nombre d'actions pour aider les jeunes. Elle est devenue le partenaire incontournable de 
la municipalité nantaise. Les effets du discrédit ont donc été levés à l'échelle locale ${ }^{16}$.

\section{Conclusion}

Qu'il s'agisse des rapports de classe, des rapports sociaux de sexe ou des rapports de racisation, les rapports sociaux de domination s'accompagnent toujours d'une décrédibilisation des membres des catégories dominées. Mais les multiples effets concrets de ce discrédit systématique, qui rendent leurs actions si difficiles et simultanément si invisibles, non seulement restent imperceptibles à tous ceux qui ne les vivent pas, mais induisent chez eux de fausses représentations. Car, dès lors qu'on ne perçoit pas les effets du discrédit, comment ne pas attribuer les échecs répétés des cours d'action des personnes en situation dominée et discréditée à leur incompétence personnelle, à leur incapacité à construire des projets réalistes, des plans stratégiques et à les mener au bout? Comment ne pas être conduit à présupposer chez eux une certaine absence de détermination, une incapacité constitutive à élaborer des plans stratégiques, un déficit de compétences pour mener à bien un cours d'action, une certaine désorganisation mentale, par opposition à la clairvoyance, à la volonté, à la confiance en soi dont font preuve par contraste les membres des catégories non touchées par cette déconsidération?

Pour ne pas charger la barque on attribuera avec bienveillance ces déficits à leur manque de capitaux. Le professeur de Julien et de Mohammed, constatant que l'un a réussi à trouver très vite le stage idéal et que l'autre n'a rien trouvé, sera conduit à attribuer faussement la réussite du premier et l'échec du second à une différence de compétence, d'activité, voire d'intelligence. Mais en fait, c'est Mohammed qui a été le plus actif, le plus volontaire, le plus déterminé, le plus réflexif.

De même, un père qui, malgré de forts vents contraires, aura obtenu à l'arraché la garde de son enfant, ne sera pas considéré pour autant comme un héros; en revanche les autres pères seront considérés - du moins dans les années 1990 - comme se désintéressant de leurs enfants : pères irresponsables, incompétents, mauvais pères. II aura fallu l'action collective du Mouvement de la condition paternelle, fédération d'associations militantes, pour renverser la situation.

16. Cf. DELCROIX, Catherine, "Discrédit et action collective. La lutte d'une association de pères musulmans", in : COSSÉE, Claire ; LADA, Emmanuelle ; RIGONI, Isabelle, Faire figure d'étranger. Regards croisés sur la production de l'altérité, Paris : Éd. Armand Colin, 2004, pp. 191-210. 
Le déni de compétence et de volonté qui colle à la peau des victimes d'un discrédit collectif est un stéréotype qui leur fait très mal. C'est une représentation totalement erronée, car les victimes sont en réalité non pas moins mais plus actives, non pas moins mais plus réflexives, non pas moins mais plus volontaires et déterminées : c'est sur ce point que nous voudrions conclure.

Bien entendu nous ne prétendons pas que les personnes qui se trouvent en situation de discrédit sont toutes plus actives, plus énergiques, plus déterminées et plus réflexives que les autres. Ce que nous avons voulu montrer ici, c'est que pour réussir à lever ce discrédit, elles le deviennent en raison même des contraintes que leur situation fait peser sur elles. En particulier celles qui ont des enfants, comme ces parents immigrés ou ces mères célibataires et pauvres que nous avons rencontrés dans nombre de cités HLM. C'est leur situation qui les force à réfléchir aux moyens d'en sortir, à chercher des informations auxquelles elles n'ont pas - au contraire d'autres - naturellement accès, à développer leurs réseaux de relations qui sont donnés d'emblée à d'autres, à réfléchir aux façons d'utiliser les informations et leurs contacts pour parvenir à atteindre leurs modestes objectifs, malgré le discrédit qui les frappe et leur attribue un déni général de compétence.

Certaines personnes finissent par plier sous le poids des difficultés, et ce sont seulement celles-là, découragées par les échecs répétés de leurs efforts, que le sens commun retient comme exemples de la véracité de ses représentations négatives. Mais d'autres continuent, se durcissent et utilisent leurs expériences récurrentes du discrédit pour affiner leurs stratégies.

En réalité, l'expertise acquise au cours d'efforts répétés constitue, pour ceux et celles qui en ont l'expérience, une ressource que l'on ne trouve guère ailleurs dans la société. 